\title{
Preparation, Characterization and Drug-Release Behaviors of Crosslinked Chitosan/Attapulgite Hybrid Microspheres by a Facile Spray-Drying Technique
}

\author{
Qin Wang ${ }^{1}$, Jie Wü ${ }^{2}$, Wenbo Wang ${ }^{1}$, Aiqin Wang, \\ ${ }^{1}$ Center of Eco-material and Green Chemistry, Lanzhou Institute of Chemical Physics, Chinese Academy of Sciences, Lanzhou, \\ China; ${ }^{2}$ Key Laboratory for Attapulgite Science and Applied Technology of Jiangsu Province, Huaiyin Institute of Technology, \\ Huaian, China. \\ Email: *aqwang@licp.cas.cn
}

Received February 28 ${ }^{\text {th }}, 2011$; revised March 20 ${ }^{\text {th }} 2011$; accepted April $20^{\text {th }}, 2011$.

\begin{abstract}
A series of chitosan/attapulgite (CTS/APT) hybrid microspheres were prepared by a facile spray-drying technique. The developed hybrid microspheres were characterized by Fourier transform infrared spectra (FTIR), X-ray powder diffraction (XRD), scanning electron microscopy (SEM) and the zeta potential. The encapsulation efficiency and in vitro controlled release properties of the microspheres for drug were evaluated using diclofenac sodium (DS) as a model drug. Results indicated that the introduction of APT into crosslinked CTS microspheres can achieve narrow size distribution and make them more uniform. The isoelectric point of the microspheres increased from 8.14 to 9.18 with increasing the content of APT to $10 \mathrm{wt} \%$. DS loaded in hybrid microspheres is hardly released in simulated gastric fluid, but quickly released in simulated intestinal fluid. The electrostatic interaction between hybrid microspheres and DS can improve the encapsulation efficiency and controlled release behavior of CTS/APT microspheres, and the release mechanism fits Fickian diffusion.
\end{abstract}

Keywords: Microsphere, Chitosan, Attapulgite, Spray Drying, Controlled Release

\section{Introduction}

During recent decades, microspheres have been widely used in pharmaceutical applications due to their numerous advantages such as higher bioavailability, prolonging drug release, high dispersibility and fast permeability as well as providing an optimal microenvironment for effective drug delivery [1]. Microspheres are mainly produced by different technological procedures like emulsion-crosslinking [2], ionic gelation [3], sieve method [4] and spray-drying technique [5,6], etc. Among them, the spray-drying technique is a one-step constructive process, which provides greater control over particle size, particle morphology and powder density and avoids tedious processes. Hence, spray-drying becomes the most facile and preferred technique for the fabrication of microspheres, and it has been used widely in medical industry due to the low cost and available equipment [7].

Chitosan (CTS) is one of the most extensively studied polysaccharides and has shown great potential as a drug carrier [8-10]. CTS microspheres obtained by spraydrying technique draw considerable attention for both oral and systemic applications in the drug delivery system. These drug-loading microspheres not only have a desirable size range in favor of respiration and absorption, but also can provide a high loading efficiency [6,11]. However, the burst effect of drug loaded in pure CTS microspheres is undesirable in neutral or basic conditions, which limited the application of CTS-based drug carriers [12-14].

Recently, polymer with layered silicate or polymer/layered silicate composites have received significant attentions from both the academic and industrial area. The introduction of clay minerals into organic matrix not only solved the burst problem of the loaded drugs, but also enhanced the stability of the delivery system in simulated gastric fluid [15-17]. Attapulgite (APT) is a 
type of naturally occurred nano-scale fibrous silicate clay, consisting of two double chains of the pyroxene-type $\left(\mathrm{SiO}_{3}\right)^{2-}$ like amphibole $\left(\mathrm{Si}_{4} \mathrm{O}_{11}\right)^{6-}$ running parallel to the fibre axis, and used in drug delivery system [18]. To date, the application of polymer/clay microspheres prepared by a spray-drying technique drug as a delivery system has not been reported. So, it is expected that the network structure and drug-delivery properties of the microspheres could be improved by the introduction of nanoscale APT into crosslinked CTS microspheres.

In this work, APT was introduced into crosslinked CTS microspheres to derive hybrid microspheres in order to alleviate the burst problem of pure CTS microspheres for DS in the gastrointestinal environment. The resultant microsphere was characterized by Fourier transform infrared spectra (FTIR), X-ray powder diffraction (XRD) and scanning electron microscopy (SEM). The encapsulation efficiency and in vitro release behaviors were systematically evaluated using diclofenac sodium (DS) as a model drug.

\section{Materials and Methods}

\subsection{Materials}

Chitosan (CTS, degree of deacetylation is 0.90 , average molecular weight is $90 \times 10^{4}$ ) was supplied by Zhejiang Yuhuan Ocean Biology Co. (Zhejiang, China). Attapulgite (APT) was supplied by Mingguang Colloidal Co. (Anhui, China). Diclofenac sodium (DS) was purchased from Jiuzhou pharmaceutical factory (He'nan, China). All the other reagents used were of analytical grade and all solutions were prepared with distilled water.

\subsection{Preparation of Crosslinked CTS/APT Hybrid Microspheres}

The CTS/APT hybrid microspheres were prepared by a spray drying technique as follows. First, CTS $(1.0 \mathrm{~g})$ was dissolved in $100 \mathrm{~mL} 1 \mathrm{w} / \mathrm{w} \%$ acetic acid solution, and a calculated amount of APT was dispersed into the solution with a continuous stirring at $200 \mathrm{rpm}$ for $4 \mathrm{~h}$ to get a homogenous dispersion. Second, a moderate amount of crosslinking agent glutaraldehyde was added into the above mixture and was further stirred at $1000 \mathrm{rpm}$ for 30 min at room temperature. The resultant suspension was then spray-dried (Mini Spray Dryer, Type SY1600, China) through a $0.7 \mathrm{~mm}$ nozzle at a feed rate of $12 \mathrm{~mL} / \mathrm{min}$, and the inlet temperature was controlled at $150^{\circ} \mathrm{C}$. Third, the yellow hybrid microsphere power can be obtained, and then dried to constant weight in an oven at $70^{\circ} \mathrm{C}$. A series of crosslinked CTS/APT hybrid microspheres with different composition were prepared as shown in Table 1.

Copyright (C) 2011 SciRes.

\subsection{Preparation of DS-Loaded Hybrid Microspheres and Determination of Encapsulation Efficiency}

$0.2 \mathrm{~g}$ DS was dissolved in $100 \mathrm{~mL}$ distilled water and then $1.0 \mathrm{~g}$ of hybrid microsphere was dispersed in the above solution. After shaking for $12 \mathrm{~h}$ in a thermostatic shaker bath (THZ-98A) set at $150 \mathrm{rpm}$ and $30^{\circ} \mathrm{C}$, DSloaded hybrid microspheres were isolated by centrifugation and then dried to constant weight in an oven at $70^{\circ} \mathrm{C}$.

To evaluate the amount of the DS entrapped inside the hybrid microspheres, an indirect method was used [19]. Aliquots from the filtered solutions remaining after the removal of the hybrid microspheres were assayed using a UV-vis spectrophotometer (SPECORD 200, ANALYTIK JENA AG) at $280 \mathrm{~nm}$. The encapsulation efficiency of DS entrapped was calculated according to the following equation:

Encapsulation efficiency $(\%)=(w-c v) \times 100 / w$

where $w$ is the mass of DS used for drug loading, $\mathrm{g} ; c$ is the concentration of DS in the filtrate $\left(\mathrm{g} \mathrm{mL}^{-1}\right)$ and $v$ is the volume of the filtrate $(\mathrm{mL})$.

\subsection{In Vitro Drug Release}

In vitro release experiments were carried out using an intelligent dissolution apparatus by immersing $0.25 \mathrm{~g}$ of the dried DS-loaded hybrid microspheres in $500 \mathrm{~mL}$ dissolution media. The dissolution media ( $\mathrm{pH} 2.1$ or $\mathrm{pH} 6.8$ ) were prepared by combining $\mathrm{HCl}, \mathrm{KH}_{2} \mathrm{PO}_{4}$ and $\mathrm{NaOH}$ solution according to the Chinese Pharmacopoeia 2005. The mixture was stirred at $50 \mathrm{rpm}$ and kept at $37 \pm 1^{\circ} \mathrm{C}$. At predetermined time intervals, $5 \mathrm{~mL}$ of the solution was taken and the same amount of the fresh dissolution media was added back to maintain a constant volume. The collected $5 \mathrm{~mL}$ of solution was filtrated through a membrane with a pore diameter of $0.45 \mu \mathrm{m}$. The filtrate $(3 \mathrm{~mL})$ was diluted to $25 \mathrm{~mL}$ with the fresh dissolution media. The concentration of DS was determined by a UV-vis spectrophotometer at the wavelength $280 \mathrm{~nm}$, and then the cumulative release percentage of DS was obtained.

\subsection{Characterization}

FTIR spectra of CTS/APT hybrid microspheres were recorded on a Thermo Nicolet NEXUS TM spectrophotometer using a $\mathrm{KBr}$ pellet. SEM observations were taken using scanning electron microscopy (JSM-5600LV, JEOL, Ltd.) after the samples were dispersed in ethanol and coated by a gold film. Power XRD analyses were performed using a diffractometer with $\mathrm{Cu}$ anode (PAN alytical X'pert PRO), running at $40 \mathrm{kV}$ and $30 \mathrm{~mA}$, scanning from $3^{\circ}$ to $40^{\circ}$ at $3^{\circ} / \mathrm{min}$. The zeta potential was measured 
by Zetasizer Nano ZS (Malvern Instruments Ltd., Worcestershire, UK) after the samples $(0.1 \mathrm{~g})$ were dispersed in $10 \mathrm{ml}$ distilled water. Main particle size was determined using OMEC LS-pop (6) laser particle analyzer (Omec technology Ltd., Zhuhai, China) after the samples $(0.1 \mathrm{~g})$ were dispersed in $2 \mathrm{ml}$ ethanol.

\subsection{Statistical Analysis}

All the tests including measurements of drug content and in vitro drug cumulative release properties were carried out three times and the averages were reported. Statistical data analysis was performed using the Student's t-test with $p<0.05$ as the minimal level of significance.

\section{Results and Discussion}

\subsection{Encapsulation Efficiency of Crosslinked CTS/APT Hybrid Microspheres for DS and Their Sizes}

Table 1 lists composition of prepared formulations, encapsulation efficiency and mean particle size of the hybrid microspheres. It can be seen that the encapsulation efficiency of the hybrid microspheres for DS is always higher than $98 \%$. This may be mainly attributed to electrostatic interaction between the microsphere carriers and the DS drug. In order to prove the electrostatic interaction, the zeta potentials of the hybrid microspheres and DS drug were determined at various $\mathrm{pHs}$ and are showed in Figure 1. It is shown that the isoelectric points of crosslinked CTS, CTS/APT-1 and CTS/APT-2 microspheres are pHs 8.14, 9.18 and 8.28, respectively; while the isoelectric point of DS is $\mathrm{pH} 4.03$. This indicates that the hybrid microspheres show positive charge, and DS possesses negative charge in distilled water ( $\mathrm{pH}$ 6.85). Therefore, a strong electrostatic interaction between the hybrid microspheres and DS leads to the higher encapsulation efficiency of the hybrid microspheres for DS.

Drug carrier systems with particle size below $200 \mu \mathrm{m}$ can prolong the gastrointestinal transit time and control the release of the encapsulated drug. But in fact, the op- timal particle size for prolonging residence time of microspheres in the colon is between 4 and $15 \mu \mathrm{m}$ [20]. From Table 1, it is observed that the mean particle sizes of the hybrid microspheres DS loaded range from $3.70 \pm$ 0.001 to $5.27 \pm 0.007 \mu \mathrm{m}\left(\mathrm{D}_{50}\right)$. This result suggests that CTS/APT hybrid microspheres can prolong and control the DS release in the colon. Moreover, microspheres in that size range are also able to attach more efficiently to the mucus layer and accumulate in the inflamed region without the need for macrophage uptake [21].

The size distribution of the hybrid microspheres was investigated and is presented in Figure 2. As can be seen, the size distribution of crosslinked CTS microspheres changes from 0.2 to $19.5 \mu \mathrm{m}$, but from 0.2 to $16.11 \mu \mathrm{m}$ for CTS/APT-1 hybrid microsphere and from 0.2 to $13.31 \mu \mathrm{m}$ for CTS/APT-2 hybrid microsphere. This indicates that the introduction of APT into crosslinked CTS microsphere can achieve narrow size distribution and make them more uniform. In addition, the particle size distribution is also expressed in terms of SPAN factor determined as:

$$
\text { SPAN }=\left(\mathrm{D}_{90}-\mathrm{D}_{10}\right) / \mathrm{D}_{50}
$$

where $\mathrm{D}_{10}, \mathrm{D}_{50}$ and $\mathrm{D}_{90}$ are the diameter sizes and the given percentage value is the percentage of particles smaller than that size. A high SPAN value indicates a wide size distribution and a high polydispersity [22]. According to Gottlieb and Schwartzbach's report [23], a relative SPAN less than 2.00 for spray-dried with $\mathrm{D}_{50}$ below $10 \mu \mathrm{m}$, is normally considered as a narrow distribution in spray drying. It was seen from Table $\mathbf{1}$ that all SPAN factor values are lower than 2.00 , indicating that CTS/APT hybrid microspheres have narrow size distribution.

\subsection{FTIR Spectra}

To prove the interaction among crosslinked CTS, APT and DS, the FTIR spectra of DS, APT, crosslinked CTS, CTS/APT-1 and DS-loaded CTS/APT-1 hybrid microspheres are shown in Figure 3. As can be seen from

Table 1. Composition of prepared formulations, encapsulation efficiency and mean particle sizes of the hybrid microspheres.

\begin{tabular}{|c|c|c|c|c|c|c|c|c|}
\hline \multirow{2}{*}{ Samples } & \multirow{2}{*}{ CTS (g) } & \multirow{2}{*}{ APT $(g)$} & \multirow{2}{*}{$\begin{array}{c}\text { Glutaraldehyde } \\
(\mathrm{mL})\end{array}$} & \multirow{2}{*}{$\begin{array}{l}\text { Encapsulation } \\
\text { Efficiency (\%) }\end{array}$} & \multicolumn{4}{|c|}{ Mean particle size $(\mu \mathrm{m})$} \\
\hline & & & & & $\mathrm{D}_{10}$ & $\mathrm{D}_{50}$ & $\mathrm{D}_{90}$ & SPAN \\
\hline Crosslinked CTS & 4.0 & 0.0 & 3.0 & $98.97 \pm 0.01$ & $2.33 \pm 0.057$ & $5.27 \pm 0.007$ & $8.82 \pm 0.156$ & $1.23 \pm 0.021$ \\
\hline CTS/APT-1 & 4.0 & 0.4 & 3.0 & $99.04 \pm 0.01$ & $2.11 \pm 0.035$ & $4.56 \pm 0.003$ & $7.47 \pm 0.092$ & $1.18 \pm 0.030$ \\
\hline CTS/APT-2 & 4.0 & 0.8 & 3.0 & $99.05 \pm 0.02$ & $2.35 \pm 0.007$ & $4.77 \pm 0.028$ & $7.49 \pm 0.085$ & $1.09 \pm 0.013$ \\
\hline CTS/APT-3 & 4.0 & 0.4 & 2.0 & $98.35 \pm 0.04$ & $2.50 \pm 0.001$ & $4.92 \pm 0.001$ & $7.57 \pm 0.014$ & $1.03 \pm 0.003$ \\
\hline CTS/APT-4 & 4.0 & 0.4 & 4.0 & $98.87 \pm 0.01$ & $1.92 \pm 0.007$ & $3.70 \pm 0.001$ & $5.64 \pm 0.007$ & $1.00 \pm 0.004$ \\
\hline
\end{tabular}




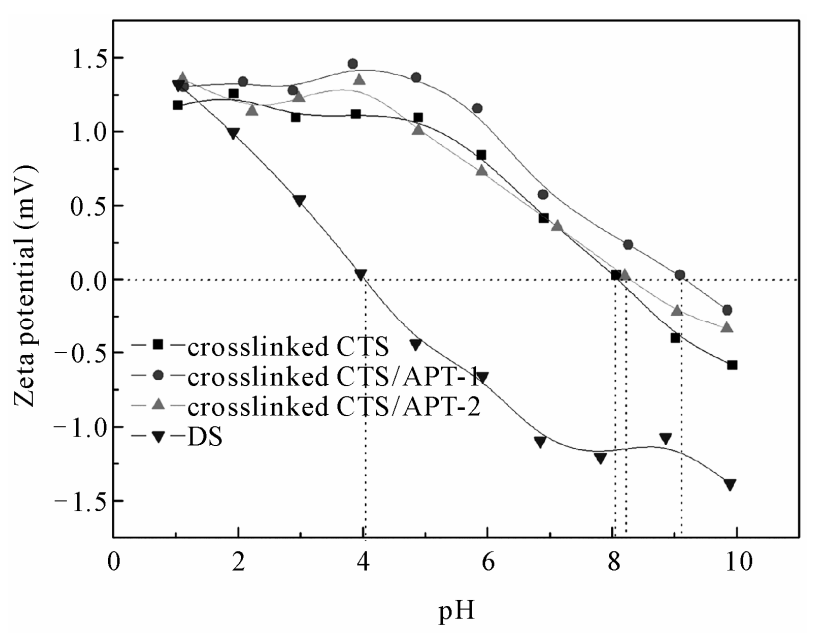

Figure 1. Values of zeta potential (mV) for crosslinked CTS, CTS/APT-1, CTS/APT-2 and DS in different pH solutions.

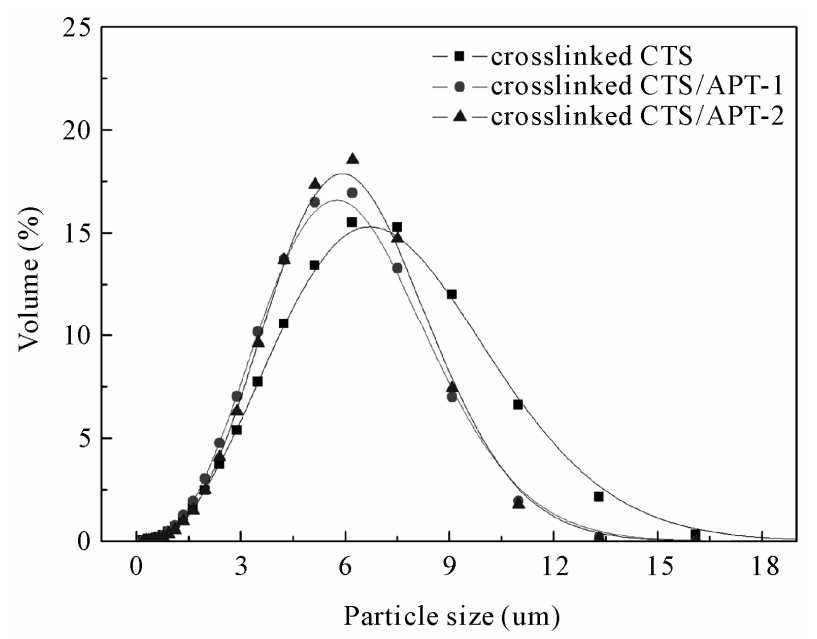

Figure 2. Particle size distribution of hybrid microspheres containing DS.

Figure 3 (b), the main absorption peaks of APT at 1027 and $982 \mathrm{~cm}^{-1}$ are ascribed to the stretching vibration of $\mathrm{Si}-\mathrm{OH}$ groups. In Figure 3 (c), the absorption peaks at $1655 \mathrm{~cm}^{-1}$ and $1560 \mathrm{~cm}^{-1}$ are observed and ascribed to the formation of imine group after crosslinking CTS with glutaraldehyde [24]. After incorporating APT into the crosslinked CTS microsphere, the FTIR spectrum in Figure 3 (d) has no change compared with that shown in Figure 3 (c). This may be because the absorption peaks of APT can be covered by the absorption peaks of crosslinked CTS. The information obtained from (b), (d) in Figure 3 indicates that APT may be dispersed into the crosslinked CTS networks by physical mixing.

FTIR spectrum of DS shows a characteristic peak at $1576 \mathrm{~cm}^{-1}$ due to the aromatic stretching and the $-\mathrm{COO}$ asymmetric stretching; the peaks observed at 1507 and $1453 \mathrm{~cm}^{-1}$ are due to N-H deformation and C-N stretching, respectively; and a sharp peak observed at $765 \mathrm{~cm}^{-1}$ is due to the $\mathrm{C}-\mathrm{Cl}$ group attached to the aromatic moiety (Figure 3 (a)). These peak positions are in accordance with the previous report [25]. The FTIR spectrum in Figure 3 (e) shows principal peaks of DS molecule at $1575,1505,1452$ and $750 \mathrm{~cm}^{-1}$ when the DS molecule were incorporated into the hybrid microspheres, which indicates the successful entrapment of DS. In addition, no extra peaks can be seen, indicating there is no chemical reaction of DS either with crosslinked CTS or with APT.

\subsection{XRD Analysis}

To further study the interaction between the crosslinked CTS and APT, the XRD patterns and typical diffraction peaks of APT, crosslinked CTS, CTS/APT-1 and CTS/ APT-2 hybrid microspheres are shown in Figure 4. As can be seen, the XRD pattern of APT in Figure 4 (a) shows a reflection peak at about $2 \theta=8.43^{\circ}$ (basal spacing $d=1.05 \mathrm{~nm}$ ), and the XRD pattern of the crosslinked CTS microspheres in Figure 4 (b) shows a reflection peak at about $2 \theta=19.17^{\circ}(d=0.46 \mathrm{~nm})$. After $10 \mathrm{wt} \%$ APT was incorporated, the typical diffraction peaks of APT (Figure 4 (c)) are greatly weakened and shifted from $8.43^{\circ}$ to $8.39^{\circ}$, and the typical diffraction peaks of the crosslinked CTS shifted from $19.17^{\circ}$ to $19.65^{\circ}$. Moreover, these diffraction peaks in Figure 4 (d) are further shifted to $8.38^{\circ}$ and $19.74^{\circ}$ as $20 \mathrm{wt} \%$ APT was introduced. However, these basal spacing corresponding in Figures 4 (a)-(d) has no change. The weaken and slight shift of these diffraction peaks of crosslinked CTS and APT may be only because of the electrostatic interaction between crosslinked CTS and APT, instead of chemical reaction of APT with crosslinked CTS. Moreover, the invariant basal spacing of APT indicates that crosslinked CTS may not be intercalated into the monolayer structure of APT.

\subsection{Morphological Analysis}

To show the influence of APT on the surface structure of crosslinked CTS microspheres more directly, the surface morphologies of crosslinked CTS/APT hybrid microspheres with different amounts of APT were also observed by SEM and are shown in Figure 5. As can be seen, the hybrid microspheres with different amounts of APT obtained by the spray-drying technique all exhibited regular spherical shape. Comparing Figure 5 (a) with Figure 5 (b) and Figure 5 (c), it can be observed that the crosslinked CTS microsphere only shows a smooth surface, whereas a coarse surface can be observed for the 


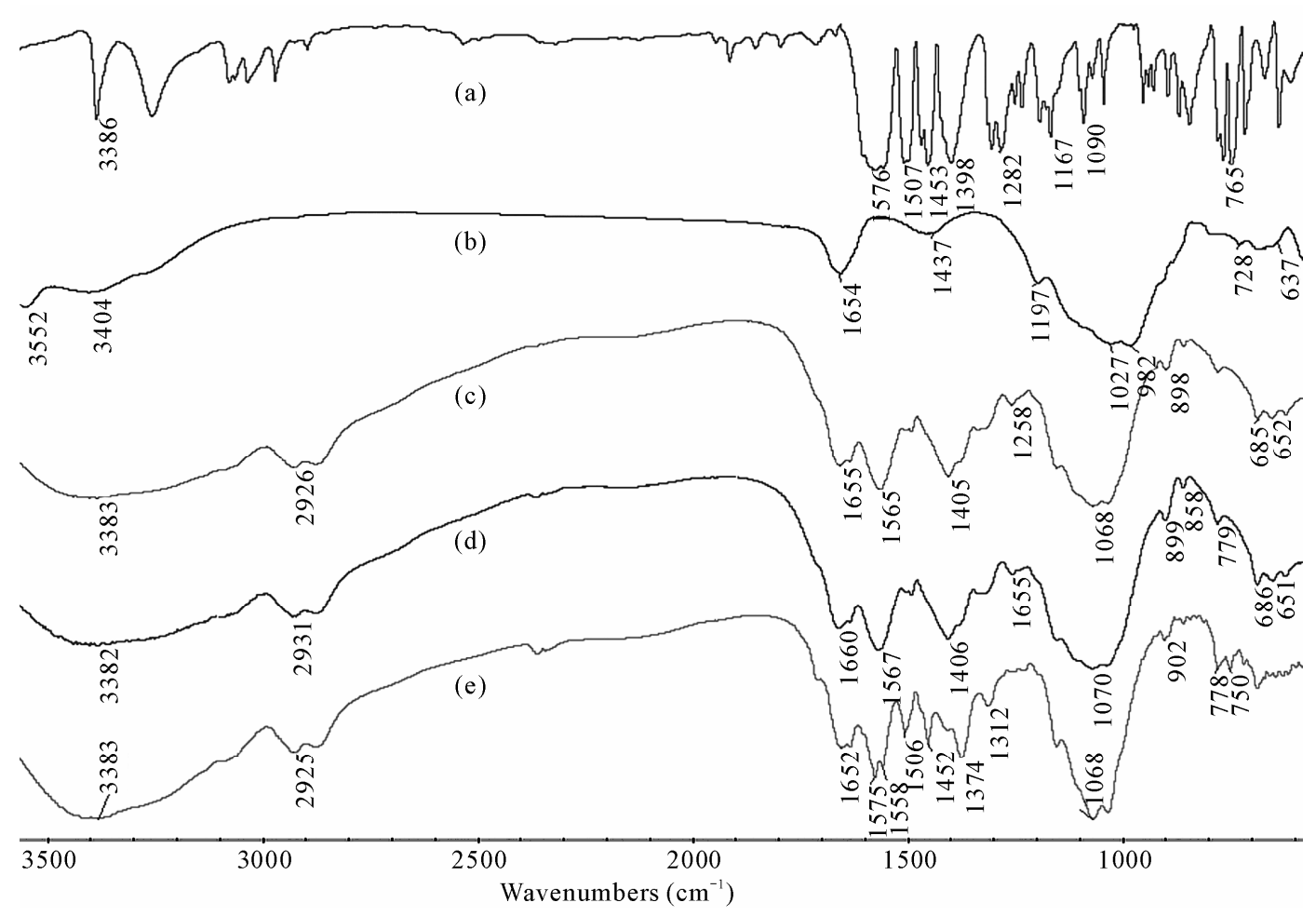

Figure 3. FTIR spectra of (a) DS, (b) APT, (c) crosslinked CTS, (d) CTS/APT-1 and (e) DS-loaded CTS/APT-1.

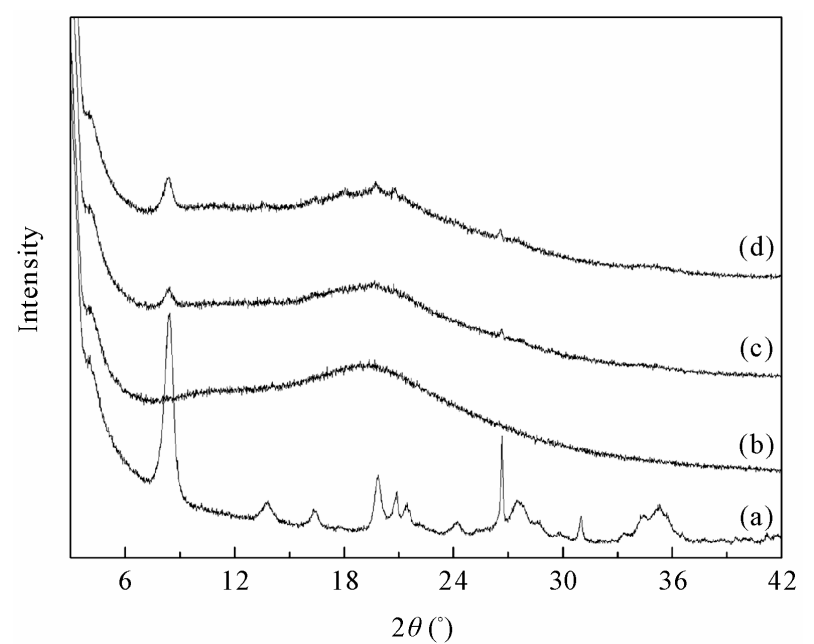

Figure 4. XRD patterns of (a) APT, (b) crosslinked CTS, (c) CTS/APT-1 and (d) CTS/APT-2.

crosslinked CTS/APT hybrid microsphere. With the further magnification of the microspheres (Figure 5(a')c')), it is found that the virgulate crystals of APT are uniformly dispersed in the microsphere, and the number of the virgulate crystals increase with enhancing the content of APT. However, the sphericity of the microsphere with higher APT content is worse than that of crosslinked CTS microsphere.

\subsection{DS Release Behaviors}

The release behaviors of DS from the microspheres with various composition were investigated and compared in simulated gastric fluid and simulated intestinal fluid. The cumulative release percentage of DS from the microsphere formulations were plotted as a function of time and are shown in Figure 6. Obviously, only little DS releases from the matrices in simulated gastric fluid due to the insolubility of DS in acidic medium. However, after being transferred into simulated intestinal fluid, the release rate is initially higher due to the dissolution of surface-adhered drug, and then the drug release rate was slowed due to the diffusion process of DS from the internal network to external solution.

In addition, the release ratio of three microspheres with various amounts of APT at $\mathrm{pH} 6.8$ phosphate buffer solution (PBS) is in the order of CTS/APT-2 > crosslinked CTS $>$ CTS/APT-1 at the same time. This may be attributed to the follow facts: 1) as can be seen from Figure 1, CTS/APT-1 hybrid microsphere has the highest positive Zeta potential at $\mathrm{pH}$ 6.8 PBS compared with crosslinked CTS and CTS/APT-2 microspheres. This indicates that crosslinked CTS/APT-1 hybrid microsphere possesses more positive charges and can produce stronger electrostatic interaction with negatively charged DS in $\mathrm{pH}$ 6.8 PBS. Therefore, the release of DS from 


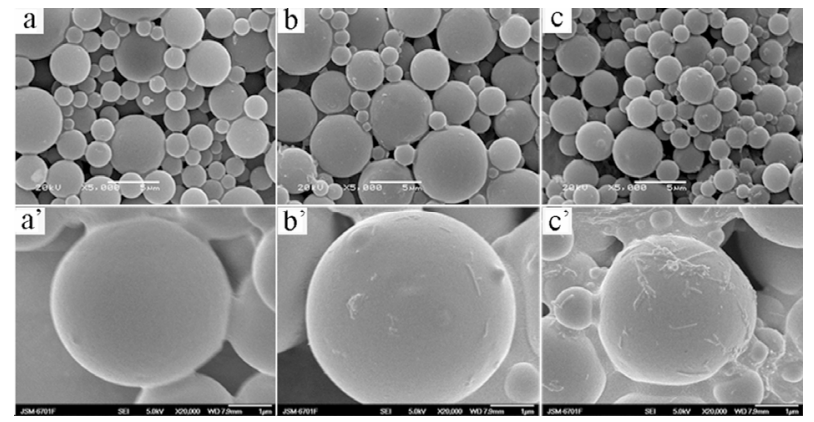

Figure 5. SEM of (a) crosslinked CTS $(\times 5000)$, (b) CTS/ APT-1 $(\times 5000)$ and (c) CTS/APT-2 $(\times 5000)$; (a') crosslinked CTS $(\times 20000)$, (b') CTS/APT-1 $(\times 20000)$ and (c') CTS/ APT-2 (×20000).

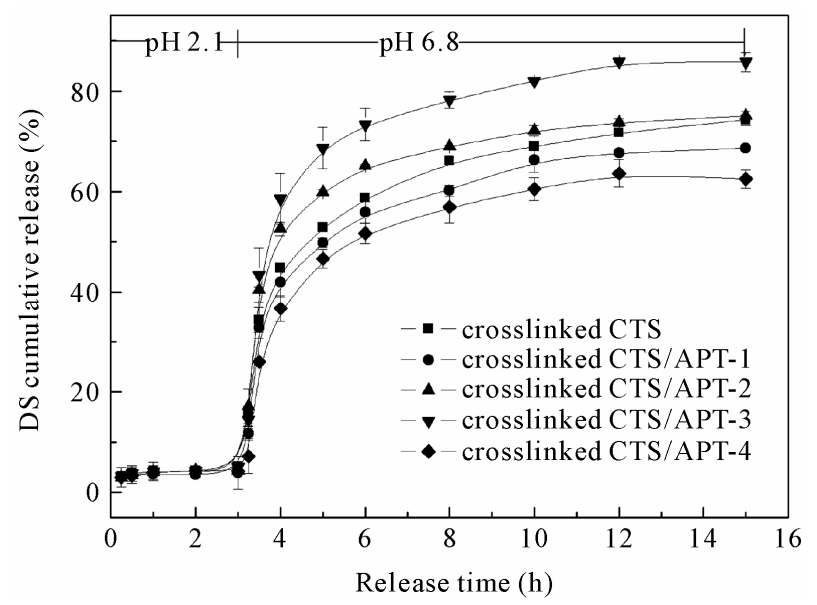

Figure 6. The cumulative release of DS from hybrid microspheres.

CTS/APT-1 hybrid microspheres is slow under the action of strong electrostatic interaction; 2) there is also electrostatic interaction between crosslinked CTS/APT-2 hybrid microspheres and DS. However, the higher content of APT causes the more coarse surface compared with crosslinked CTS and CTS/APT-1 microsphere (Figure 5), and the coarse surface makes DS deposit on the surface of the hybrid microsphere in the process of DS adsorption and microsphere washing. As a result, the marked burst effect of DS from the surface of crosslinked CTS/ APT-2 hybrid microsphere is observed.

As can be also seen from Figure 6, the CTS/APT-3 hybrid microspheres shows the highest drug release ratio, followed by CTS/APT-1 and CTS/APT-4 at the same time (the concentration of glutaraldehyde is different). CTS contains large amounts of chemically reactive $-\mathrm{NH}_{2}$ and $-\mathrm{OH}$ groups, and its free amino groups can react with glutaraldehyde to form a crosslinked polymeric network. But a lower glutaraldehyde concentration can only generate a looser network structure and allows faster diffu- sion of the loaded drug into the dissolution media [26]. Therefore, the release rate of DS from the hybrid microspheres decreases significantly with increasing the concentration of glutaraldehyde.

In order to further understand the release behavior of DS from the hybrid microspheres in $\mathrm{pH}$ 6.8 PBS, in vitro release data in Figure 6 were fitted to various models to analyze the kinetics and mechanism of DS release from the hybrid microspheres. For elucidating the kinetics of DS release, the experimental data was analyzed using zero-order Equation (3), first-order Equation (4) [27,28], higuchi's square root model Equation (5) [29], and the Power law Equation (6) [30,31]. Where, $F_{t}$ is the drug release fraction at time " $t$ ", $k_{1-4}$ is the release constant of respective equations, $t$ is the release time, and $n$ is the characteristic diffusion exponent.

$$
\begin{gathered}
F_{t}=k_{1} t \\
F_{t}=1-e_{2}^{-k t} \\
F_{t}=k_{3} t^{1 / 2} \\
F_{t}=k_{4} t^{n}
\end{gathered}
$$

The correlation coefficient $\left(r_{4}^{2}\right)$ for Equation (6) is between 0.988 and 0.97 for the microspheres released at pH 6.8 PBS (Table 2). This is correspondingly higher than the other kinetic equations, namely Zero-order, First-order kinetics and Higuchi equation, suggesting that the release mode of DS follows the Power law. In many experimental situations, including the case of drug release from the microsphere system, the mechanism of DS diffusion deviates from the Fickian equation and follows a non-Fickian (anomalous) behavior. For a sphere, when $n<0.43$, this represents a Fickian diffusion mechanism. When $n>0.86$, this represents a Case II transport mechanism. When $n$ value is between 0.43 and 0.86 , the transport is a typical and both Fickian diffusion and Case II transport contribute to the release, i.e. anomalous transport. In these cases, the calculated values of $n$ by Power law for Equation (6) are shown in Table 2. The results of $n$ presented in Table 2 are in the range of 0.160 $\sim 0.227$. This demonstrates that the release mechanism fits Fickian diffusion.

\section{Conclusions}

In this work, DS-loaded CTS/APT hybrid microspheres with controlled release properties in the simulated intestinal fluid were prepared by a facile spray-drying technique. The results from FTIR spectra and XRD patterns showed that APT was dispersed into hybrid microspheres by physical mixing and DS is stable in the matrices developed without undergoing any chemical changes. In 
Table 2. The release mechanism of DS from hybrid microspheres.

\begin{tabular}{|c|c|c|c|c|c|c|c|c|c|c|}
\hline \multirow{2}{*}{ Sample } & \multicolumn{2}{|c|}{ Zero-order } & \multicolumn{2}{|c|}{ First-order } & \multicolumn{2}{|c|}{ Higuchi } & \multicolumn{3}{|c|}{ Power law } & \multirow{2}{*}{ Release mechanism } \\
\hline & $k_{1}$ & $r_{1}^{2}$ & $k_{2}$ & $r_{2}^{2}$ & $k_{3}$ & $r_{3}^{2}$ & $n$ & $k_{4}$ & $r_{4}^{2}$ & \\
\hline Crosslinked CTS & 0.033 & 0.884 & 0.083 & 0.933 & 0.139 & 0.960 & 0.227 & 0.447 & 0.988 & Fickian diffusion \\
\hline CTS/APT-1 & 0.029 & 0.878 & 0.073 & 0.920 & 0.124 & 0.949 & 0.203 & 0.456 & 0.978 & Fickian diffusion \\
\hline CTS/APT-2 & 0.026 & 0.893 & 0.068 & 0.944 & 0.111 & 0.961 & 0.162 & 0.531 & 0.988 & Fickian diffusion \\
\hline CTS/APT-3 & 0.026 & 0.871 & 0.113 & 0.948 & 0.112 & 0.948 & 0.143 & 0.603 & 0.983 & Fickian diffusion \\
\hline CTS/APT-4 & 0.031 & 0.869 & 0.062 & 0.916 & 0.131 & 0.949 & 0.260 & 0.358 & 0.97 & Fickian diffusion \\
\hline
\end{tabular}

addition, the introduction of APT can not only enhance the isoelectric points but also achieve narrow size distribution of hybrid microspheres, which made the hybrid microspheres exhibit slow and sustained drug release profiles in the simulated intestinal fluid due to the stronger electrostatic interaction and smaller average sizes. Based on all experimental results, it can be concluded that CTS/APT hybrid microspheres loaded with DS could be suitable candidates for oral delivery of DS with controlled release properties, opening a new therapeutic potential for this carriers for local treatment of chronic inflammatory and degenerative joint diseases.

\section{Acknowledgements}

The authors would like to thank the "Special Research Fund of Scholarship of Dean of CAS" and Jiangsu Provincial Science and Technology Office (No.BE2009098) for financial support of this research.

\section{REFERENCES}

[1] S. Freiberg and X. X. Zhu, "Polymer Microspheres for Controlled Drug Release," International Journal of Pharmaceutics, Vol. 282, No. 1-2, 2004, pp. 1-18. doi:10.1016/j.ijpharm.2004.04.013

[2] R. H. Qin, F. S. Li, M. Y. Chen and W. Jiang, "Preparation of Chitosan-Ethylene Diaminetetraacetate-Enwrapped Magnetic $\mathrm{CoFe}_{2} \mathrm{O}_{4}$ Nanoparticles via Zero-Length Emulsion Crosslinking Method," Applied Surface Science, Vol. 256, No. 1, 2009, pp. 27-32. doi:10.1016/j.apsusc.2009.07.032

[3] A. R. Dudhani and S. L. Kosaraju, "Bioadhesive Chitosan Nanoparticles: Preparation and Characterization," Carbohydrate Polymers, Vol. 81, No. 2, 2010, pp. 243-251. doi:10.1016/j.carbpol.2010.02.026

[4] S. A. Agnihotri and T. M. Aminabhavi, "Controlled Release of Clozapine Through Chitosan Microparticles Prepared by a Novel Method," Journal of Controlled Release, Vol. 96, No. 2, 2004, pp. 245-259. doi:10.1016/j.jconrel.2004.01.025

[5] D. Chiou and T. A. G. Langrish, "Development and Characterisation of Novel Nutraceuticals with Spray Drying
Technology," Journal of Food Engineering, Vol. 82, No. 1, 2007, pp 84-91. doi:10.1016/j.jfoodeng.2007.01.021

[6] L. Ochiuz and J. E. Peris, "Preparation and Characterisation of Alendronate-Loaded Chitosan Microparticles Obtained through the Spray Drying Technique," Medicinal Chemistry, Vol. 5, No. 2, 2009, pp. 191-196.

[7] P. Tewa-Tagne, S. Briançon and H. Fessi, "Preparation of Redispersible Dry Nanocapsules by Means of Spray- Drying: Development and Characterization," European Journal of Pharmaceutical Sciences, Vol. 30, No. 2, 2007, pp. 124-135. doi:10.1016/j.ejps.2006.10.006

[8] K. Vimala, M. M. Yallapu, K. Varaprasad, N. N. Reddy, S. Ravindra, N. S. Naidu and K. M. Raju, "Fabrication of Curcumin Encapsulated Chitosan-PVA Silver Nanocomposite Films for Improved Antimicrobial Activity," Journal of Biomaterials and Nanobiotechnology, Vol. 2, No. 1, 2011, pp. 55-64.

[9] H. Li, C. R. Zhou, J. H. Tian and J. H. Rong, "Preparation and Characterization of Homogeneous Hydroxyapatite/Chitosan Composite Scaffolds via In-Situ Hydration," Journal of Biomaterials and Nanobiotechnology, Vol. 1, No. 1, 2010, pp. 42-49. doi:10.4236/jbnb.2010.11006

[10] Y. Murata, D. Hirai, K. Kofuji, E. Miyamoto and S. Kawashima, "Properties of an Alginate Gel Bead Containing a Chitosan-Drug Salt," Biological \& Pharmaceutical Bulletin, Vol. 27, No. 3, 2004, pp. 440-442. doi: $10.1248 / \mathrm{bpb} .27 .440$

[11] P. He, S. S. Davis and L. Illum, "Chitosan Microspheres Prepared by Spray Drying," International Journal of Pharmaceutics, Vol. 187, No. 1, 1999, pp. 53-65. doi:10.1016/S0378-5173(99)00125-8

[12] P. Giunchedi, C. Juliano, E. Gavini, M. Cossu and M. Sorrenti, "Formulation and in Vivo Evaluation of Chlorhexidine Buccal Tablets Prepared Using Drug-Loaded Chitosan Microspheres," European Journal of Pharmaceutics and Biopharmaceutics, Vol. 53, No. 2, 2002, pp. 233-239. doi:10.1016/S0939-6411(01)00237-5

[13] D. O. Corrigan, A. M. Healy and O. I. Corrigan, "Preparation and Release of Salbutamol from Chitosan and Chitosan Co-spray Dried Compacts and Multiparticulates," European Journal of Pharmaceutics and Biopharmaceutics, Vol. 62, No. 3, 2006, pp. 295-305. doi:10.1016/i.ejpb.2005.09.008 
[14] A. Alhalaweh, S. Andersson and S. P. Velaga, "Preparation of Zolmitriptan-Chitosan Microparticles by Spray Drying for Nasal Delivery," European Journal of Pharmaceutical Sciences, Vol. 38, No. 3, 2009, pp. 206-214. doi:10.1016/j.ejps.2009.07.003

[15] J. P. Zhang, Q. Wang and A. Q. Wang, "In situ Generation of Sodium Alginate/ Hydroxyapatite Nanocomposite Beads as Drug-Controlled Release Matrices," Acta Biomaterialia, Vol. 6, No. 2, 2010, pp. 445-454. doi:10.1016/j.actbio.2009.07.001

[16] M. D. Arco, E. Cebadera, S. Gutiérrez, C. Martín, M. J. Montero, V. Rives, J. Rocha and M. A. Sevilla, "Mg, Al Layered Double Hydroxides with Intercalated Indomethacin: Synthesis, Characterization, and Pharmacological Study," Journal of Pharmaceutical Science, Vol. 93, No. 6, 2004, pp. 1649-1658. doi:10.1002/jps.20054

[17] Q. Wang, X. L. Xie, X. W. Zhang, J. P. Zhang and A. Q. Wang, "Preparation and Swelling Properties of $\mathrm{Ph}$ Sensitive Composite Hydrogel Beads Based on Chitosan-GPoly (Acrylic Acid)/Vermiculite and Sodium Alginate for Diclofenac Controlled Release," International Journal of Biological Macromolecules, Vol. 46, No. 3, 2010, pp. 356-362. doi:10.1016/j.ijbiomac.2010.01.009

[18] M. P. S. Krekeler and S. Guggenheim, "Defects in Microstructure in Palygorskite-Sepiolite Minerals: A Transmission Electron Microscopy (TEM) Study," Applied Clay Science, Vol. 39, No. 1-2, 2008, pp. 98-105. doi:10.1016/j.clay.2007.05.001

[19] M. J. Fernández-Hervás, M. A. Holgado, A. Fini and J. T. Fell, "In Vitro Evaluation of Alginate Beads of Diclofenac Salts", International Journal of Pharmaceutics, Vol. 163, No. 1-2, 1998, pp. 23-34. doi:10.1016/S0378-5173(97)00333-5

[20] A. Lamprecht, N. Ubrich, H. Yamamoto, U. Schäfer, H. Takeuchi, P. Maincent, Y. Kawashima and C. M. Lehr, "Biodegradable Nanoparticles for Targeted Drug Delivery in Treatment of Inflammatory Bowel Disease," The Journal of Pharmacology and Experimental Therapeutics, Vol. 299, No. 2, pp. 775-781.

[21] A. Lamprecht, U. Schafer and C. M. Lehr, "Size-Dependent Bioadhesion of Micro- and Nanoparticulate Carriers to the Inflamed Colonic Mucosa," Pharmaceutical Research, Vol. 18, No. 6, pp. 788-793. doi:10.1023/A:1011032328064

[22] R. R. Dubey and R. H. Parikh, "Studies of PLGA Microspheres," Pharmaceutical Technology Europe, Vol. 16, 2004, pp. 23-34.

[23] N. Gottlieb and C. Schwartzbach, "Development of an Internal Mixing Two-Fluid Nozzle by Systematic Variation of Internal Parts," Proceedings of the Americas: In- stitute for Liquid Atomization and Spray Systems Conference, Nottingham, 16-19 May 2004.

Http://www.niro.com/niro/cmsresources.nsf/filenames/TF N_Niro_Ilass_2004_Final.pdf/\$file/TFN_Niro_Ilass_200 4_Final.pdf

[24] V. H. Kulkarni, P. V. Kulkarni and J. Keshavayya, "Glutaraldehyde-Crosslinked Chitosan Beads for Controlled Release of Diclofenac Sodium," Journal of Applied Polymer Science, Vol. 103, No. 1, 2007, pp. 211-217. doi:10.1002/app.25161

[25] T. Iliescu, M. Baia and W. Kiefer, "FT-Raman, SurfaceEnhanced Raman Spectroscopy and Theoretical Investigations of Diclofenac Sodium," Chemical Physics, Vol. 298, No. 1-3, 2004, pp. 167-174. doi:10.1016/j.chemphys.2003.11.018

[26] F. L. Mi, C. Y. Kuan, S. S. Shyu, S. T. Lee and S. F. Chang, "The Study of Gelation Kinetics and Chain-Relaxation Properties of Glutaraldehyde-Cross-Linked Chitosan Gel and Their Effects on Microspheres Preparation and Drug Release," Carbohydrate Polymers, Vol. 41, No. 4, 2000, pp. 389-396. doi:10.1016/S0144-8617(99)00104-6

[27] M. Gibaldi and S. Feldman, "Establishment of Sink Conditions in Dissolution Rate Determinations-Theoretical Considerations and Application to Nondisintegrating Dosage Forms," Journal of Pharmaceutical Sciences, Vol. 56, No. 10, 1967, pp. 1238-1242. doi:10.1002/jps.2600561005

[28] J. G. Wagner, "Interpretation of Percent Dissolved-Time Plots Derived from in Vitro Testing of Conventional Tablets and Capsules," Journal of Pharmaceutical Sciences, Vol. 58, No. 10, 1969, pp. 1253-1257. doi: $10.1002 / j p s .2600581021$

[29] T. Higuchi, "Mechanism of Sustained-Action Medication. Theoretical Analysis of Rate of Release of Solid Drugs Dispersed in Solid Matrices," Journal of Pharmaceutical Sciences, Vol. 52, No. 12, 1963, pp. 1145-1149. doi:10.1002/jps.2600521210

[30] P. L. Ritger and N. A. Peppas, "A Simple Equation for Description of Solute Release. I. Fickian and Non-Fickian Release from Non-Swellable Devices in the Form of Slabs, Spheres, Cylinders or Discs," Journal of Controlled Release, Vol. 5, No. 1, 1987, pp. 23-36. doi:10.1016/0168-3659(87)90034-4

[31] J. Siepmann and N. A. Peppas, "Modeling of Drug Release from Delivery Systems Based on Hydroxypropyl Methylcellulose (HPMC)," Advanced Drug Delivery Reviews, Vol. 48, No. 2-3, 2001, pp. 139-157. doi:10.1016/S0169-409X(01)00112-0 